\title{
Transthoracic delivery of large devices into the left ventricle through the right ventricle and interventricular septum: preclinical feasibility
}

Majdi Halabi', Kanishka Ratnayaka1,3, Anthony Z Faranesh', Michael S Hansen ${ }^{1}$, Israel M Barbash', Michael A Eckhaus², Joel R Wilson', Marcus Y Chen ${ }^{1}$, Michael C Slack³, Ozgur Kocaturk', William H Schenke', Victor J Wright ${ }^{1}$ and Robert J Lederman ${ }^{1 *}$

\begin{abstract}
Background: We aim to deliver large appliances into the left ventricle through the right ventricle and across the interventricular septum. This transthoracic access route exploits immediate recoil of the septum, and lower transmyocardial pressure gradient across the right versus left ventricular free wall. The route may enhance safety and allow subxiphoid rather than intercostal traversal.
\end{abstract}

Methods: The entire procedure was performed under real-time CMR guidance. An "active" CMR needle crossed the chest, right ventricular free wall, and then the interventricular septum to deliver a guidewire then used to deliver an $18 \mathrm{Fr}$ introducer. Afterwards, the right ventricular free wall was closed with a nitinol occluder. Immediate closure and late healing of the unrepaired septum and free wall were assessed by oximetry, angiography, CMR, and necropsy up to four weeks afterwards.

Results: The procedure was successful in 9 of 11 pigs. One failed because of refractory ventricular fibrillation upon needle entry, and the other because of inadequate guidewire support. In all ten attempts, the right ventricular free wall was closed without hemopericardium. There was neither immediate nor late shunt on oximetry, X-ray angiography, or CMR. The interventricular septal tract fibrosed completely. Transventricular trajectories planned on human CT scans suggest comparable intracavitary working space and less acute entry angles than a conventional atrial transseptal approach.

Conclusion: Large closed-chest access ports can be introduced across the right ventricular free wall and interventricular septum into the left ventricle. The septum recoils immediately and heals completely without repair. A nitinol occluder immediately seals the right ventricular wall. The entry angle is more favorable to introduce, for example, prosthetic mitral valves than a conventional atrial transseptal approach.

Keywords: Catheterization, Interventional cardiovascular MR, Structural heart disease

\section{Background}

Implanting large appliances into the heart, such as investigational transcatheter mitral or aortic valves, will require large introducer sheaths. Transfemoral arterial access may be limited by vessel caliber or disease [1]; transvascular retrograde aortic access risks atheroembolism and stroke

\footnotetext{
* Correspondence: lederman@nih.gov

${ }^{1}$ Cardiovascular and Pulmonary Branch, Division of Intramural Research, NHLBI, NIH, Building 10, Room 2c713, MSC 1538, Bethesda, MD 20892-1538, USA

Full list of author information is available at the end of the article
}

[2]. Minithoracotomy access to the left ventricular (LV) apex $[3,4]$ or ascending aorta [5] provides a "straight shot" for large rigid devices, but requires surgical repair. Transcatheter access across the interatrial septum creates unfavorable entry angles and working space [6,7]. A subxiphoid route into the left ventricle would allow delivery of large and rigid implants if introducers could enter and exit gracefully.

We have proposed closed-chest direct transthoracic closure of large access ports directly across the LV free wall using nitinol cardiac closure devices [8], and of

\section{Biomed Central}


direct transthoracic closure of ventricular septal defect across the right ventricular free wall [9]. We noticed while attempting to create an animal model of muscular ventricular septal defect, that aggressive dilatation without ablation of the interventricular septum failed to induce a persistent defect or shunt [9]. Moreover, knife injury across the interventricular septum often fails to induce persistent myocardial defects once hemopericardium is treated [10,11]. Liu and colleagues [12] report a different surgical approach to the LV across the RV free wall and interventricular septum in animals.

These observations led us to propose an alternative closed-chest large-port access route intended (1) to allow entry across the mitral valve orifice; (2) to exploit the reduced leakage pressure gradient across the RV free wall to allow safe closure compared with leakage pressure across the LV free wall; (3) to test the immediate recoil and late healing of large unrepaired sheath ports across the interventricular septum; and (4) to allow a subxiphoid rather than intercostal route.

We hypothesize that $18 \mathrm{Fr}$ sheaths can be introduced into beating LV in animals successively across the chest wall, RV free wall, and interventricular septum; that the interventricular septum closes immediately and thereafter fibroses completely; and that the large RV free wall hole can be closed with a nitinol closure device intended to seal atrial septal defect. We perform these procedures in swine using real-time CMR guidance to provide global imaging context of the transthoracic procedure.

\section{Methods}

\section{Animals}

Animal procedures were approved by the institutional animal care and use committee according to contemporary $\mathrm{NIH}$ guidelines. The study was performed in 11 naïve Yorkshire pigs $(49 \pm 6 \mathrm{~kg})$, after a period of technical development in separate animals. Anesthesia was induced with ketamine, midazolam and glycopyrrolate and maintained with inhaled isoflurane and mechanical ventilation. For survival experiments, percutaneous catheters (see below) were removed and animals provided a fentanyl patch and ketoprofen analgesia as needed.

\section{Catheters}

We customized an 18 G CMR-compatible nitinol needle to enhance its visibility during CMR [13]. By adding solenoid receiver coils along the shaft of the needle, we created an antenna that can be connected to the CMR scanner as a receive coil. This "active" antenna detects a signal that can be reconstructed on the CMR scanner on a separate channel. We depict "active" devices in color (in this case, green) overlaid upon the greyscale tissue image, allowing the user to recognize a specific imaging appearance of the device [14].

After accessing the LV cavity, off-the-shelf stiff nitinol wires (0.038"Angled Stiff Shaft Glidewire, Terumo Medical Corporation or Nitrex Guidewire, EV3 Inc.) were used to exchange sequentially for an 8 Frx $10 \mathrm{~cm}$ short introducer sheath (Pinnacle introducer sheath, Terumo) and then a custom $18 \mathrm{Fr}$ introducer sheath. We customized a commercial introducer (Large Check-Flo, Cook Medical, Bloomington, IN) to shorten the sheath from 30 to $15 \mathrm{~cm}$, and we designed and printed a dilator lock to secure it to the sheath hub (P450 ABS modeling material, Uprint rapid prototype printer, Stratsys, Eden Prairie, MN), shown in Figure 1.

At the conclusion of procedures, the RV free wall entry site was closed using an off-the-shelf nitinol atrial septal occluder (Amplatzer Septal Occluder, courtesy of St Jude Medical, St. Paul, MN). We modified the delivery cable to incorporate a loopless antenna to enhance visibility during CMR [15].

\section{CMR}

The CMR suite (1.5 T Espree Siemens, Germany) is configured for interventional guidance as described previously [8], and as shown in Figure 2. The $70 \mathrm{~cm}$ scanner bore allows chest access. A commercial prototype workstation (Interactive Front End, Siemens) connected to the CMR scanner provides a graphic user interface for interactive slice and scan control and low-latency reconstruction of real-time multislice CMR. The prototype is further customized with NHLBI features including interactive slice thickness, interactive projection mode, and interactive saturation magnetization preparation. In-lab LCD projectors display scanner control, real-time imaging, and instantaneous hemodynamics. Operators wear

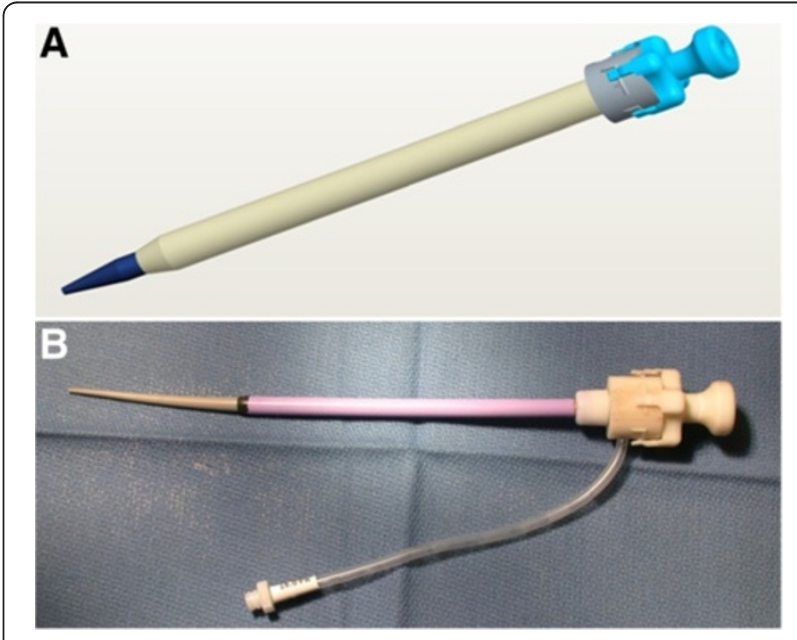

Figure 1 The $18 \mathrm{Fr}$ sheath is modified by shortening (to $15 \mathrm{~cm}$ ) and using a 3D-printed locking dilator (A, blue). 


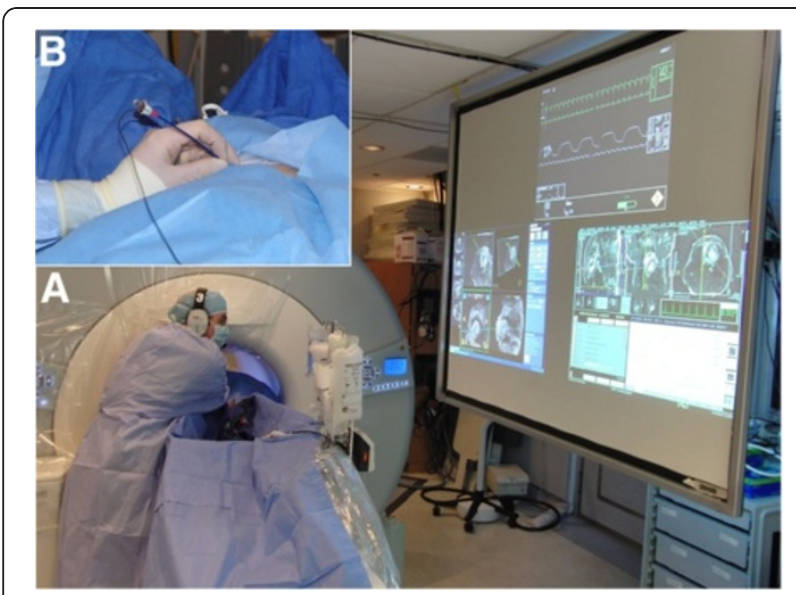

Figure 2 The interventional CMR environment used here. Panel (A) shows the operator leaning into the $70 \mathrm{~cm}$ bore to establish transthoracic access during real-time CMR. LCD projectors display instantaneous hemodynamics, scanner control, and CMR. Acoustic noise suppression headsets allow communication with staff in the control room. Inset (B) shows the access port with the table moved out of the scanner, viewed from the left leg.

acoustic noise cancellation headsets and microphones to communicate all at once to team members in- and outside the laboratory (IMROC, Optoacoustics, Moshav Mazor, Israel).

Anatomy and function scans used ECG-gated, segmented breath-hold balanced steady-state free precession (bSSFP) CMR, as well as late gadolinium enhancement using gadopentetate-dimeglumine $0.2 \mathrm{mmol} / \mathrm{kg}$. Typical real-time CMR parameters were: bSSFP; FOV, $350 \mathrm{~mm}$; slice thickness, $6 \mathrm{~mm}$; TR/TE, $2.88 / 1.44 \mathrm{~ms}$; flip angle 40 degrees, matrix, $192 \times 144$, parallel imaging rate 2 , temporal resolution $242 \mathrm{~ms}$ per slice. Images were constructed and projected on a screen inside the MR suite for the operators.

High resolution ex-vivo CMR examined the transseptal tract scar. 3-dimensional T1-weighted spoiled gradient echo achieved $0.6 \mathrm{~mm}$ isotropic voxel resolution using TR/TE, 10.0/5.35 ms; 8 averages, 100-120slices per slab; flip angle, $20^{\circ}$; matrix, 320×320×144; FOV, $200 \mathrm{~mm}$.

\section{Interventional procedure}

Separate non-survival experiments were performed in 9 animals to explore initial techniques. Early failure modes included: inadequate skin incision to allow large-sheath entry; and inadequate guidewire support to deliver the 18Fr introducer sheath. Thereafter we performed prospective survival experiments on 11 additional animals reported here.

At baseline, a subxiphoid pericardial drain was placed at baseline to instill fluid to separate the pericardial walls and allow appropriate positioning of the epicardial disc of the nitinol closure device ("permissive pericardial tamponade") [16]. Percutaneous sheaths were placed into the femoral artery and vein. Baseline LV and RV angiography, and pulmonary artery catheterization were performed with oximetry.

CMR guided three key procedure steps. First, a transthoracic needle trajectory was selected. Considerations include avoiding interposed bony structures and intercostal spaces, minimal interposed lung, and a pathway from the RV free wall towards the interventricular septum that enters the mid-LV cavity without papillary muscle injury or entrapment. The trajectory was planned interactively using a finger on the chest surface, and images were stored as a roadmap for subsequent CMR slice prescription. It is important to note that while we are modeling a subxiphoid procedure for human use, the rotation of the porcine heart requires a right lateral transthoracic trajectory in these experiments.

Next the needle was advanced percutaneously during real-time CMR using two interleaved long-axis images for guidance. The shallow needle trajectory is ergonomically suitable for both animal and operator inside the CMR bore. LV cavity entry was confirmed with imaging and pressure measurements before a passive $0.035 " \times$ $150 \mathrm{~cm}$ nitinol guidewire (Angled Standard Glidewire, Terumo, Somerset, NJ) was used to place a small (8Fr $\times$ $10 \mathrm{~cm}$ ) introducer sheath. Next an air-filled balloon wedge endhole catheter (7Fr, Arrow-Teleflex) was exchanged into the LV and manipulated into a pulmonary vein or the descending aorta to achieve safe guidewire purchase for the next step. Next a stiff 0.038 " nitinol guidewire (Angled Stiff Glidewire, Terumo) delivered a large 18Fr introducer port modified as described above (Catheter Devices).

No implant was delivered during these experiments, which test access and closure only. The final step is withdrawal of the port and closure of the RV free wall entry site. Because the $18 \mathrm{Fr}$ sheath has no discrete imaging characteristics, the balloon wedge endhole catheter was used in tandem with the sheath to depict its tip during withdrawal into the RV. A coaxial 8 Frx $23 \mathrm{~cm}$ sheath was used to position the nitinol closure device across the RV free wall. $100-150 \mathrm{~mL}$ of saline was injected via the separate pericardial catheter, lowering the blood pressure $\sim 25 \%$, in order to separate the pericardial walls and allow the proximal (epicardial) disc to be deployed along the epicardial border.

\section{Follow-up}

Follow-up was designed to detect early or late hemopericardium, iatrogenic myocardial dysfunction, and to characterize the extent of myocardial injury caused by the trans-ventricular access tract.

After RV closure the animals were observed for two hours, before follow-up CMR and catheter angiographic and oximetric assessment. 
Late CMR follow-up was performed on day 4 and week 4, including cine CMR for function and pericardial effusion, and late gadolinium enhancement for interventricular septum inspection. Animals were euthanized after four weeks and the heart extracted for high resolution ex-vivo late gadolinium enhanced (3D T1-weighted) CMR. Necropsy and histopathology by a dedicated veterinary pathologist evaluated the presence of ventricular septal defect or scar.

\section{Trajectory planning in humans}

We assessed the anatomic feasibility of transthoracic interventricular septal access in humans using an anonymized and de-linked series of adults with suspected cardiovascular disease undergoing contrast-enhanced computed tomography $(\mathrm{CT}, 320 \times 0.5-\mathrm{mm}$ detector rows, Aquillion One, Toshiba Medical Systems, Japan) extending to the abdomen using ECG-gated breath-held acquisitions (courtesy of M.Y. Chen, NHLBI DIR). These do not constitute human subject research under US 45CFR $\$ 46.102(f)$.

\section{Data analysis}

LV mass, function, scar volume, and trajectory geometries were measured using a CMR workstation (Leonardo, Siemens). Paired continuous parameters were compared using a Student t-test. Serial continuous parameters were tested using ANOVA. A p value $<0.05$ was considered significant.

\section{Results}

Feasibility of direct RV-septal- LV access under real-time CMR guidance

After initial technique development in 9 animals, survival experiments were performed in 11 additional swine, and were successful in 9. One failed because of refractory ventricular fibrillation immediately after LV puncture. In the other, the large sheath failed to deliver because we had not yet learned to use deep guidewire support in the left atrium or aorta. This animal recovered uneventfully after RV port closure. The technique was modified in response to both (oral and intravenous amiodarone loading, and deep guidewire support after needle entry).

Figure 3 depicts a typical procedure sequence. The active needle, attached to a separate CMR receiver channel, is depicted in green during real-time CMR. In this animal, the guidewire positioned in the descending aorta couples electrically with the active needle and therefore also appears in green. After the $18 \mathrm{Fr}$ port is introduced into the LV across the interventricular septum, its tip is visualized using an air-filled balloon catheter (panel 1D) as it is repositioned into the RV. Finally the RV entry hole is sealed with a nitinol atrial septal occluder device. No therapeutic procedure is performed in this experiment.
No ventricular septal defect was evident immediately after port closure, either by oximetry (Table 1), X-ray angiography, or by CMR at 0 or 2 hours.

\section{RV free wall closure}

After the $18 \mathrm{Fr}$ (7.3 mm sheath outer diameter) port was introduced across the RV into the LV, the RV free wall hole was closed under real-time CMR guidance using an Atrial Septal Occluder 6-8 mm (St Jude Medical, Figure 3, panels E-F).

When we downsized the RV sheath from $18 \mathrm{Fr}$ to $8 \mathrm{Fr}$ to deploy the nitinol occluder, pericardial fluid leaked around the access site and failed to accumulate in 3/9 cases. As a result, the epicardial disc of the nitinol occluder failed to seat inside the pericardial space. Nevertheless, RV free wall closure was successful in 9/9 attempts, and without hemopericardium.

\section{Evaluation of the interventricular septal tract and of the myocardium}

Baseline ejection fraction as measured by CMR was $55 \pm 6 \%$ and remained unchanged after 2 hours, 4 days and 4 weeks $(\mathrm{p}=0.70)$, including among individual animals. No regional wall motion abnormality was detected.

Late gadolinium enhancement CMR in-vivo revealed a low-volume interventricular septal scar along the sheath tract. This tract was better depicted using high-resolution ex-vivo CMR (Figure 4). The calculated scar volume was $0.16 \pm 0.03 \mathrm{~g}(0.18 \% \pm 0.02$ of the LV mass $)$. Histopathology after four weeks showed a conical tract $5.1 \pm 1.3 \mathrm{~mm}$ at the RV side and $3.8 \pm 0.75 \mathrm{~mm}$ at the LV side that traversed the interventricular septum (Figure 5), with organized fibrosis. There was no evidence of ventricular septal defect. The right ventricular free wall closure device was completely endothelialized on endocardial and epicardial surfaces (Figure 6).

\section{Complications}

There were two cases of ventricular fibrillation during LV puncture or wire passage. In one, resuscitation was unsuccessful (described above), and in the other, after defibrillation the procedure continued without incident.

One animal had a large and two others small reactive (serous) pericardial effusion at 4 weeks, and one had a small right pleural effusion.

There were no cases of hemopericardium after RV free wall closure. There was no evident ECG conduction delay in any animal. There was no evident pneumothorax or lung injury.

\section{Trajectory planning in humans}

Although these porcine experiments necessarily used a right lateral transthoracic trajectory, we examined the feasibility of a subxiphoid trajectory to the LV through the RV and interventricular septum in 21 patients (13 


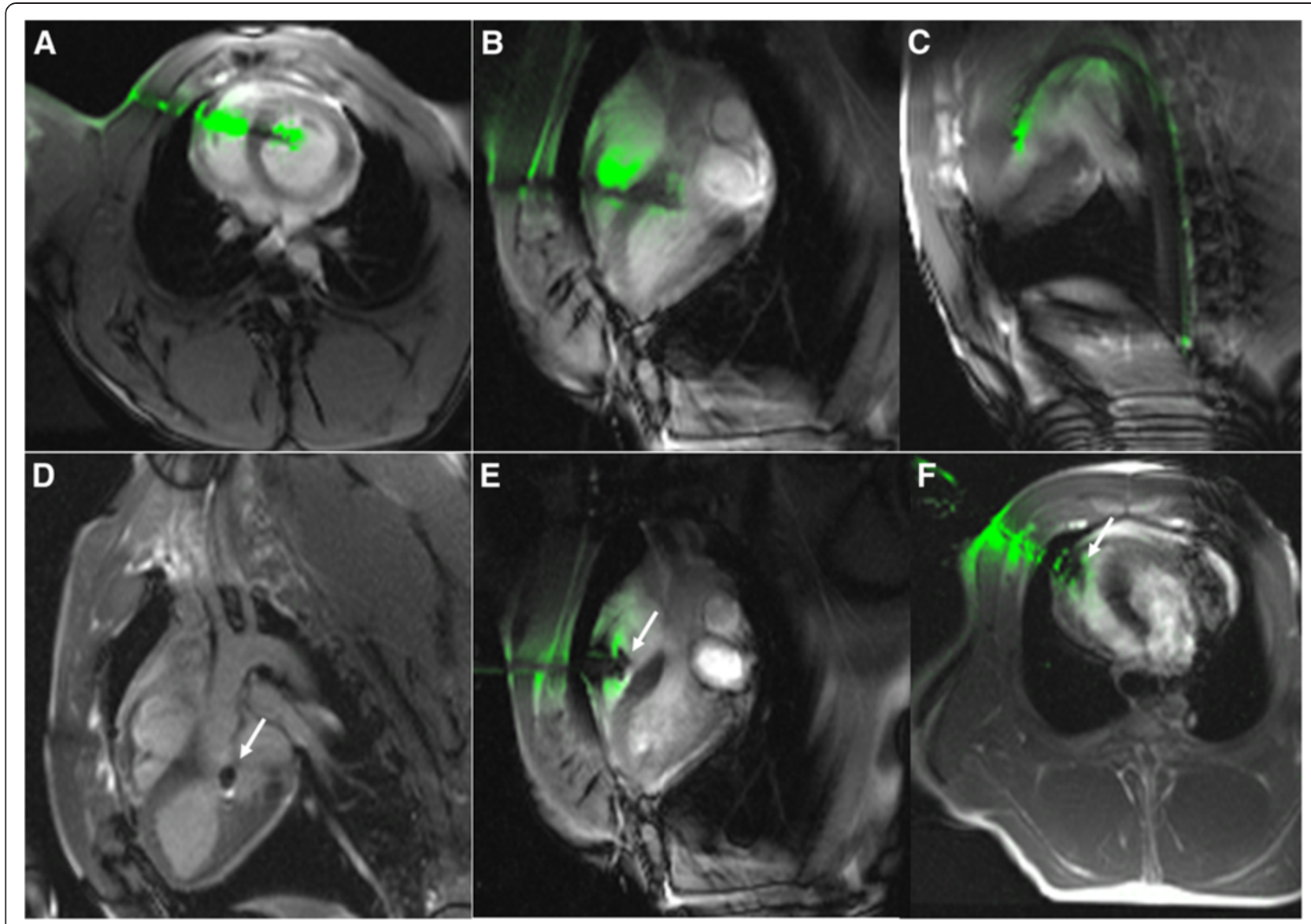

Figure 3 Real-time CMR of a typical procedure. (A,B) Simultaneous views of needle across chest and septum into the LV. (C) Guidewire through needle into descending aorta. (D) Balloon catheter indicates sheath tip (arrow) as it is withdrawn. (E, F) RV free wall closure using a nitinol occluder (arrow).

men and 8 women; mean age $52 \pm 6$ years) who underwent cardiac computed tomography with abdominal and thoracic coverage for cardiovascular disease. We measured geometry of theoretical trajectories using our novel subxiphoid-transventricular approach, and using "conventional" approach across the interatrial septum, comparing intracameral "working space" (distance) and catheter angulation (Figure 7).

The distance from the skin to the RV epicardium was $50 \pm 12 \mathrm{~mm}$. The distance from the RV epicardium to the LV endocardium through the interventricular septum was $45 \pm 6 \mathrm{~mm}$. Table 2 indicates that both

\section{Table 1 Hemodynamics and oximetry}

\begin{tabular}{lccc}
\hline & Baseline & 2 hours post procedure & p value \\
\hline Hemodynamics & & & $97 \pm 28$ \\
\hline Heart rate beats/min & $81 \pm 18$ & $51 \pm 6$ & 0.17 \\
\hline Mean arterial pressure $\mathrm{mmHg}$ & $54 \pm 10$ & & 0.50 \\
\hline Oxygen Saturation & & $95 \pm 0.7$ & 0.20 \\
\hline Femoral artery\% & $95 \pm 0.6$ & $66 \pm 7.5$ & 0.20 \\
\hline Right atrium\% & $71 \pm 6.2$ & $67 \pm 7.8$ & 0.16 \\
\hline RV\% & $72 \pm 6.7$ & $66 \pm 8.8$ & 0.14 \\
\hline Pulmonary artery\% & $72 \pm 7.1$ & $0.93 \pm 0.02$ & 0.80 \\
\hline Qp/Qs & $0.89 \pm 0.01$ & $7.0 \pm 0.7$ & 0.30 \\
\hline Hemoglobin $(\mathrm{g} / \mathrm{dL})$ & $8.4 \pm 3.9$ &
\end{tabular}




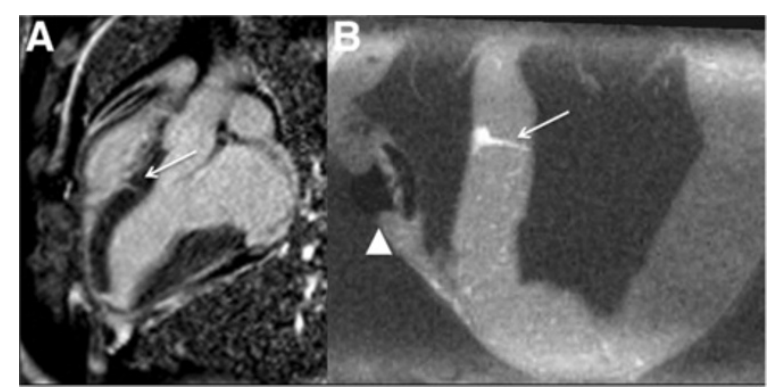

Figure 4 In-vivo (A) and high-resolution ex-vivo late-gadolinium CMR of the healed interventricular septum tract (arrow) and nitinol occluder (arrowhead) after four weeks.

transventricular and atrial transseptal approaches for transcatheter mitral valve implantation provided similar intracameral working distance between cavity entry and the target valve annulus, however our transventricular approach afforded a less acute angle $\left(148 \pm 6^{\circ}\right.$ vs $98.9 \pm$ $\left.5^{\circ}, \mathrm{p}<0.01\right)$.

\section{Discussion}

We have demonstrated a new closed-chest transthoracic access procedure into the beating LV across the interventricular septum. This approach sequentially traverses the RV free wall and then the interventricular septum instead of the LV free wall. The cavity-to-pericardial ("leakage") pressure gradient is lower for a RV free wall entry compared with LV free wall entry, and in this way our trajectory may enhance acute procedural safety of closed-chest direct cardiac access. Translated into patients, our approach would allow subxiphoid introduction of large appliances into the heart without surgery, for example transcatheter mitral valves having delivery systems $10 \mathrm{~mm}$ wide or more [17]. In this preclinical experience, we found that the entry tract across the interventricular septum recoiled completely, was associated with no early or late ventricular septal defect, was associated with no evident myocardial dysfunction or conduction abnormality, and left a small residual fibrotic

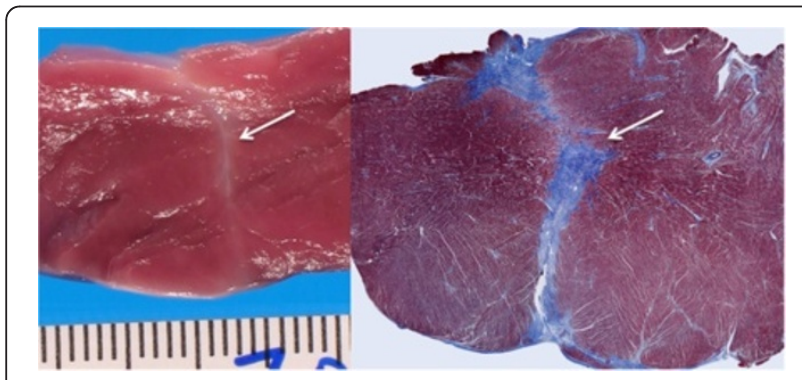

Figure 5 Fresh macroscopic and Masson trichrome (collagenavid) microscopic images of the interventricular septal tract (arrow) after four weeks, showing fibrosis and no evident bystander injury.

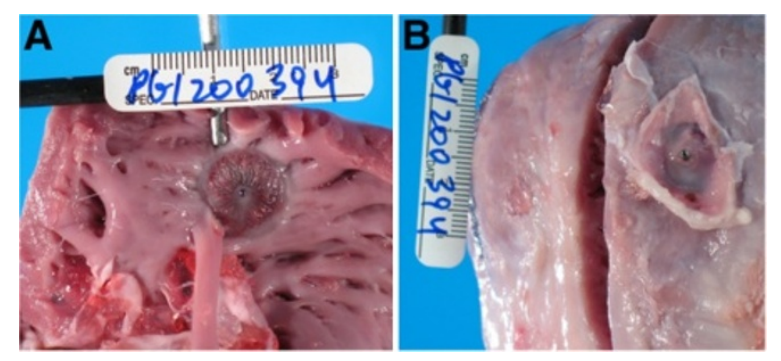

Figure 6 Macroscopic specimen of the ASD device from the RV cavity (A) and outside the RV free wall (B) four weeks after implantation. The endocardial surface is partially fibrosed.

scar with no evident bystander injury. A commercial nitinol occluder device successfully closed the RV free wall access port. Real-time CMR guidance enabled straightforward performance of this procedure by providing broad thoracic context and clear soft tissue trajectories. Our review of 21 adult human CT data sets suggests a subxiphoid transcatheter trajectory should be feasible with entry angles that are suitable for treating the aortic and mitral valves, and reduced angulation towards the mitral valve compared with a conventional atrial transseptal approach. We believe this work establishes proofof-concept for a new closed-chest non-surgical access port procedure to implant large cardiac appliances when transvascular access is not suitable.

To our surprise during the conception of this experiment, the $7.3 \mathrm{~mm}$ diameter iatrogenic ventricular septal defect recoiled acutely and then healed spontaneously and completely in every case. This is consistent with reports of spontaneous closure of traumatic ventricular septal defects, summarized by Dehgani and colleagues [11]. This also is consistent with the difficulty we experienced attempting to create an animal model of muscular ventricular septal defect without mural laser ablation [9]. Presumably the immediate recoil facilitates rapid healing, despite the large transseptal pressure gradient, manifest as a clinically-insignificant fibrotic tract after four weeks. The volume of septal scar is comparatively smaller than small CMR defects generated during otherwise uncomplicated percutaneous coronary intervention [18]. Liu and colleagues [12] report a similar "transventricular-transseptal" approach with a $5 \mathrm{~cm}$ subxiphoid incision to deliver a transcatheter aortic valve implant. They chose to close the iatrogenic ventricular septal defect with a nitinol occluder where our experience suggests this may be unnecessary.

Possible advantages of our approach compared with surgical or percutaneous transapical LV access include (1) subxiphoid access without intercostal injury and pain for transthoracic transapical access; (2) reduced cameralpericardial pressure gradient contributing to bleeding across the access tract; (3) lower likelihood of major 


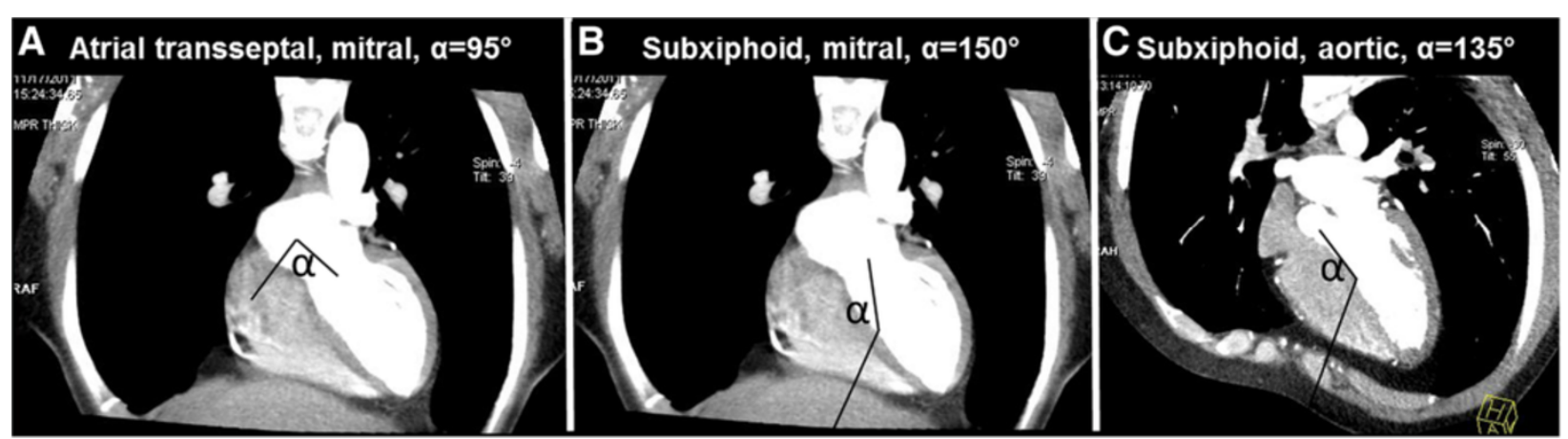

Figure 7 Theoretical trajectories from human CTA. (A) "Conventional" atrial transseptal trajectory to the mitral valve has entry angle (a) =95". (B) Subxiphoid trajectory to the mitral valve across the RV and interventricular septum, $a=150^{\circ}$. (C) Subxiphoid trajectory to the aortic valve across the RV and interventricular septum, $a=130^{\circ}$. These also depict the distances reported in Table 2.

epicardial coronary artery injury from access across the RV free wall compared with the LV apex; (4) an extrapleural and subxiphoid trajectory reducing the risk of pulmonary injury and avoiding intercostal width and trajectory constraints. Possible disadvantages of our approach include (1) injury to the interventricular septum causing a persistent ventricular septal defect or conduction abnormality, even though we observed neither in this preclinical experience; (2) shorter intracavitary "working distance" to the mitral or aortic valves compared with transapical access, and angulated entry to the aortic valve; (3) possible interference with the endocardial closure device by RV mural trabeculation, a phenomenon we did not observe in this preclinical experience; and (4).pulmonary hypertension related valve disease might reduce the proposed benefit of lower pressure gradient across the RV free wall.

There is little tolerance for risk in a transcatheter alternative to surgery. For this procedure, we have developed techniques to mitigate risk. First, a separate pericardial catheter drain is placed at the beginning of the procedure, intended to separate the pericardial walls by transient fluid installation. This drain can decompress the pericardium in an emergency. Second, an ancillary "buddy" guidewire may allow transcatheter rescue should the nitinol closure device accidentally be withdrawn through the right ventricular free wall. In our prior preclinical report of direct transthoracic repair of ventricular septal defect [9], inadvertent RV pull- through was an observed adverse event for which we proposed to apply a balloon-tipped delivery sheath. However, in the current series, there were no inadvertent RV pull-through events. Third, bailout surgical repair of RV free wall perforation is technically feasible. Finally, preliminary feasibility of device closure of cardiac free walls has been suggested by numerous clinical reports of closure of cardiac perforations using unmodified Amplatzer Septal Occluder devices [19], of transapical LV access ports up to 12Fr using Amplatzer Duct Occluders [20], or of intraoperative closure of perforations with Amplatzer Muscular VSD Occluder [21].

Real-time CMR provided superb guidance for trajectory planning, needle access, guidewire and sheath delivery, and the RV free wall closure. CMR has an advantage over X-ray or ultrasound guidance in providing softtissue images in any arbitrary orientation, independent of acoustic windows imposed by ribs or air. Most important, the large field of view provided by real-time CMR provides a global thoracic context of the procedure allowing the operator to avoid bystander tissue injury and monitor instantaneously for complications $[8,16]$.

\section{Limitations}

This work has noteworthy limitations. The procedure succeeded in only 9 of 11 animals. One failure is attributed to needle-induced ventricular fibrillation, which is common in swine. Another failure is attributed to immature catheter technique, from failure to obtain deep

Table 2 Geometry of transventricular and conventional trajectories based on human CT data

\begin{tabular}{|c|c|c|c|c|}
\hline & \multicolumn{2}{|c|}{ Transventricular LV endocardial entry across interventricular septum } & \multicolumn{2}{|c|}{ Conventional LA entry through fossa ovalis } \\
\hline & Distance (mm) & Angle & Distance (mm) & Angle \\
\hline To aortic valve annulus & $43 \pm 4$ & $125 \pm 6^{\circ}$ & - & - \\
\hline To mitral valve annulus & $44 \pm 4$ & $148 \pm 6^{\circ} *$ & $37.8 \pm 5.7$ & $98.9 \pm 5$ \\
\hline
\end{tabular}

Distances are reported between chamber entry site (LV endocardial border for transventricular access, LA border for atrial transseptal access) and the center of the target valve annulus. Angles are reported between chamber entry site and a line directly to the center of the target valve annulus. These are depicted graphically in Figure $6 .{ }^{*}=p<0.01$ compared with an atrial transseptal trajectory. 
purchase of a rigid guidewire in order to deliver the cardiac sheath. Late complications were evident in one animal, manifest as serous (reactive) pericardial effusion without signs of tamponade. In clinical practice we would expect to leave a pericardial drainage catheter in place for one or more days in an attempt to prevent pericardial effusion. Real-time CMR remains investigational, although early human CMR catheterization procedures are being undertaken $[22,23]$.

\section{Conclusions}

We have developed a novel non-surgical approach to introduce large structural implants into the LV. A subxiphoid introducer sheath is placed across the RV free wall and from there across the interventricular septum into the LV. The entry angle for mitral valve implants is less acute than a conventional atrial transseptal trajectory. The RV free wall hole is closed with a nitinol occluder device. Surprisingly, the interventricular septum hole closes immediately and completely, and afterwards heals as a small fibrous tract.

\section{Abbreviations}

ECG: Electrocardiogram; FOV: Field of view; CMR: Cardiovascular magnetic resonance; LV: Left ventricle; RV: Right ventricle; TE: Echo time; TR: Repetition time.

\section{Competing interests}

$\mathrm{NIH}$ and Siemens Medical Systems have a collaborative research and development agreement for CMR.

Kanishka Ratnayaka serves without compensation on a Siemens Pediatric Advisory Council. Michael Slack is compensated as physician proctor for St Jude Medical.

\section{Authors' contributions}

$\mathrm{MH}$ designed and performed experiments, analyzed the data, and prepared and edited the manuscript. KR, AZF, MSH, IMB JRW, MYC, MCS, OK, WHS, VJW assisted in experiments and edited the manuscript. MAE performed necropsy and histopathology analysis. RJL designed experiments, analyzed the data, and prepared and edited the manuscript. All authors read and approved the final manuscript.

\section{Acknowledgements}

Supported by the Division of Intramural Research, NHLBI, NIH (ZO1HL005062-08, Z01-HL006040-01, and Z01-HL006041-01 to RJL). We thank Kalyanam Shivkumar (UCLA) for provocative discussions; Patrick Cooke (St Jude Medical) for occluder devices; and Katherine Lucas, Joni Taylor, and Toby Rogers for technical support.

\section{Author details}

${ }^{1}$ Cardiovascular and Pulmonary Branch, Division of Intramural Research, NHLBI, NIH, Building 10, Room 2c713, MSC 1538, Bethesda, MD 20892-1538, USA. ${ }^{2}$ Division of Veterinary Resources, NIH, Bethesda, MD, USA. ${ }^{3}$ Department of Cardiology, Children's National Medical Center, Washington, DC, USA.

Received: 19 September 2012 Accepted: 14 January 2013

Published: 18 January 2013

\section{References}

1. Grube E, Buellesfeld L, Mueller R, Sauren B, Zickmann B, Nair D, Beucher H, Felderhoff T, Iversen S, Gerckens U: Progress and current status of percutaneous aortic valve replacement: results of three device generations of the CoreValve revalving system. Circ Cardiovasc Interv 2008, 1:167-175.
2. Kodali SK, Williams MR, Smith CR, Svensson LG, Webb JG, Makkar RR, Fontana GP, Dewey TM, Thourani VH, Pichard AD, et al.: Two-year outcomes after transcatheter or surgical aortic-valve replacement. $N$ Engl $J$ Med 2012, 366:1686-1695.

3. Lichtenstein SV, Cheung A, Ye J, Thompson CR, Carere RG, Pasupati S, Webb JG: Transapical transcatheter aortic valve implantation in humans: initial clinical experience. Circulation 2006, 114:591-596.

4. Seeburger J, Borger MA, Tschernich H, Leontjev S, Holzhey D, Noack T, Ender J, Mohr FW: Transapical beating heart mitral valve repair. Circ Cardiovasc Interv 2010, 3:611-612.

5. Bapat V, Khawaja MZ, Attia R, Narayana A, Wilson K, Macgillivray K, Young C, Hancock J, Redwood S, Thomas M: Transaortic transcatheter aortic valve implantation using edwards sapien valve: a novel approach. Catheter Cardiovasc Interv 2011, 79:733-740.

6. Cribier A, Eltchaninoff H, Tron C, Bauer F, Agatiello C, Sebagh L, Bash A, Nusimovici D, Litzler PY, Bessou JP, Leon MB: Early experience with percutaneous transcatheter implantation of heart valve prosthesis for the treatment of end-stage inoperable patients with calcific aortic stenosis. J Am Coll Cardiol 2004, 43:698-703.

7. Sakata Y, Syed Z, Salinger MH, Feldman T: Percutaneous balloon aortic valvuloplasty: antegrade transseptal vs. conventional retrograde transarterial approach. Catheter Cardiovasc Interv 2005, 64:314-321.

8. Barbash IM, Saikus CE, Faranesh AZ, Ratnayaka K, Kocaturk O, Chen MY, Bell JA, Virmani R, Schenke WH, Hansen MS, et al.: Direct percutaneous left ventricular access and port closure: pre-clinical feasibility. JACC CardiovasC Interv 2011, 4:1318-1325.

9. Ratnayaka K, Saikus CE, Faranesh AZ, Bell JA, Barbash IM, Kocaturk O, Reyes CA, Sonmez M, Schenke WH, Wright VJ, et al.: Closed-chest transthoracic magnetic resonance imaging-guided ventricular septal defect closure in swine. JACC Cardiovasc Interv 2011, 4:1326-1334.

10. Carr JA, Buterakos R, Bowling WM, Janson L, Kralovich KA, Copeland C, Link R, Roiter C, Casey G, Wagner JW: Long-term functional and echocardiographic assessment after penetrating cardiac injury: 5-year follow-up results. J Trauma 2011, 70:701-704.

11. Dehghani P, Ibrahim R, Collins N, Latter D, Cheema AN, Chisholm RJ: Posttraumatic ventricular septal defects-review of the literature and a novel technique for percutaneous closure. J Invasive Cardiol 2009, 21:483-487.

12. Liu L, Tozzi P, Ferrari E, von Segesser LK: The transventricular-transseptal access to the aortic root: a new route for extrapleural trans-catheter aortic stent-valve implantation. Eur J Cardiothorac Surg 2011, 39:635-641.

13. Saikus CE, Ratnayaka K, Barbash IM, Colyer JH, Kocaturk O, Faranesh AZ, Lederman RJ: MRI-guided vascular access with an active visualization needle. J Magn Reson Imaging 2011, 34:1159-1166.

14. Ratnayaka K, Faranesh AZ, Guttman MA, Kocaturk O, Saikus CE, Lederman $\mathrm{RJ}$ : Interventional cardiovascular magnetic resonance: still tantalizing. J Cardiovasc Magn Reson 2008, 10:62.

15. Bell JA, Saikus CE, Ratnayaka K, Barbash IM, Faranesh AZ, Franson DN, Sonmez M, Slack MC, Lederman RJ, Kocaturk O: Active delivery cable tuned to device deployment state: enhanced visibility of nitinol occluders during preclinical interventional MRI. J Magn Reson Imaging 2012, 36:972-978.

16. Barbash IM, Saikus CE, Ratnayaka K, Faranesh AZ, Kocaturk O, Wu V, Bell JA, Schenke WH, Raman VK, Lederman RJ: Limitations of closing percutaneous transthoracic ventricular access ports using a commercial collagen vascular closure device. Catheter Cardiovasc Interv 2011, 77:1079-1085.

17. lino K, Boldt J, Lozonschi L, Metzner A, Schoettler J, Petzina R, Cremer J, Lutter G: Off-pump transapical mitral valve replacement: evaluation after one month. Eur $J$ Cardiothorac Surg 2012, 41:512-517.

18. Locca D, Bucciarelli-Ducci C, Ferrante G, La Manna A, Keenan NG, Grasso A, Barlis P, Del Furia F, Prasad SK, Kaski JC, et al.: New universal definition of myocardial infarction applicable after complex percutaneous coronary interventions? JACC CardiovasC Interv 2010, 3:950-958.

19. Stolt V, Cook S, Raber L, Wani S, Garachamani A, Vogel R, Seiler C, Windecker S, Meier B: Amplatzer septal occluder to treat iatrogenic cardiac perforations. Catheter Cardiovasc Interv 2012, 79:263-270.

20. Jelnin V, Dudiy Y, Einhorn BN, Kronzon I, Cohen HA, Ruiz CE: Clinical experience with percutaneous left ventricular transapical access for interventions in structural heart defects a safe access and secure exit. JACC Cardiovasc Interv 2011, 4:868-874. 
21. Rahmanian PB, Filsoufi F, Castillo JG, Adams DH: Video-assisted trans-mitral left ventricular false aneurysm repair with a septal occluder.

Eur I Cardiothorac Surg 2007, 32:799.

22. Tzifa A, Krombach GA, Kramer N, Kruger S, Schutte A, von Walter M, Schaeffter T, Qureshi S, Krasemann T, Rosenthal E, et al.: Magnetic resonance-guided cardiac interventions using magnetic resonancecompatible devices: a preclinical study and first-in-man congenital interventions. Circ Cardiovasc Interv 2010, 3:585-592.

23. Ratnayaka K, Faranesh AZ, Hansen MS, Stine AM, Halabi M, Barbash IM, Schenke WH, Wright VJ, Grant LP, Kellman P, et al.: Real-time MRI-guided right heart catheterization in adults using passive catheters. Eur Heart $\rfloor$ 2012, epub 2012/08/03, PMID: 22855740.

doi:10.1186/1532-429X-15-10

Cite this article as: Halabi et al:: Transthoracic delivery of large devices into the left ventricle through the right ventricle and interventricular septum: preclinical feasibility. Journal of Cardiovascular Magnetic

Resonance 2013 15:10.

\section{Submit your next manuscript to BioMed Central} and take full advantage of:

- Convenient online submission

- Thorough peer review

- No space constraints or color figure charges

- Immediate publication on acceptance

- Inclusion in PubMed, CAS, Scopus and Google Scholar

- Research which is freely available for redistribution

Submit your manuscript at www.biomedcentral.com/submit

C BioMed Central 